\title{
Rituximab: Baustein zahlreicher Kombinationen
}

\author{
Seit 13 Jahren wird der mono- \\ klonale Anti-CD20-Antikörper \\ Rituximab beim Follikulären \\ Lymphom (FL), beim Diffus \\ Kleinzelligen B-Zell-Lymphom \\ (DLBCL) und bei der Chronisch \\ Lymphatischen Leukämie \\ (CLL) eingesetzt. In all diesen \\ Indikationen ist der Wirkstoff \\ fester Bestandteil der jewei- \\ ligen Standardtherapie.
}

Beim DLBCL, einem aggressiven Lymphom, beispielsweise konnte die Heilungsrate durch Addition von Rituximab zum
Standardregime CHOP deutlich erhöht werden, erklärte Michael Pfreundschuh, Homburg/Saar. Bei nicht vorbehandelten älteren Patienten (> 60 Jahre) sind $8 \mathrm{Zy}$ klen Rituximab und 6 Zyklen CHOP-14 (R-CHOP) die derzeit effektivste Therapie [Coiffier B. Blood 2010;116(12):2040-5]. Sie verbesserte die Rate an Komplettremissionen und das Gesamtüberleben signifikant. Unter R-CHOP überlebten $36,5 \%$ der Patienten mindestens zehn Jahre progressionsfrei, unter $\mathrm{CHOP}$ allein nur $20 \%$. In der RICOVER-Studie ließ sich durch kürzere Therapieintervalle (statt drei nur zwei Wochen) und die Hinzunahme von Rituximab in das Regime das progressionsfreie- und das Gesamtüberleben signifikant verlängern: Das 3-Jahres-Überleben betrug unter R-CHOP-14 78\%, unter CHOP-14 dagegen $68 \%$ [Pfreundschuh $\mathrm{M}$ et al. Lancet Oncol. 2008;9(2):10516]. Auch bei jüngeren DLBCLPatienten unter 60 Jahren ist mittlerweile R-CHOP Standard. ay

Symposium im Rahmen des DGHO-Kongresses 2011 am 1. Oktober 2011 in Basel, Schweiz; Veranstalter: Roche Pharma

\section{Multiples Myelom}

\section{Therapieergebnisse verbessern durch Proteasom-Inhibition}

\begin{abstract}
Beim multiplen Myelom steht eine Vielzahl von Therapieregimen und Substanzen zur Verfügung, um dem einzelnen Patienten eine möglichst effektive Behandlung zu bieten. Dem ProteasomInhibitor Bortezomib kommt hier ein hoher Stellenwert zu.
\end{abstract}

Die Kombination aus Bortezomib (Velcade ${ }^{\circ}$ ) und Prednison/ Melphalan (VMP) hat sich in der VISTA-Studie als sehr effizient für die Behandlung älterer, nicht für eine Hochdosistherapie geeig-

neter Patienten erwiesen, so Hans Salwender, Hamburg. Nach einem dreijährigen Follow-up lebten im VMP-Arm noch $68 \%$ der Patienten vs. $54 \%$ im MP-Kontrollarm. Das mediane Gesamtüber-

\section{Kurz notiert}

\section{Pemetrexed bewährt sich als Kombinationspartner bei betagten NSCLC-Patienten}

Die Zahl betagter Patienten mit fortgeschrittenen nicht plattenepithelialen nicht kleinzelligen Bronchialkarzinomen (NSCLC) wird weiter zunehmen. Das ist bei der Therapieplanung zu berücksichtigen. Der überwiegende Teil der nicht plattenepithelialen NSCLC sind Adeno- und großzellige Karzinome. Für Patienten in fortgeschrittenen Stadien dieses Tumortyps steht mit Pemetrexed (Alimta ${ }^{\oplus}$ ) plus Cisplatin eine effektive Erstlinientherapie zur Verfügung. Dies belegt eine Studie von Scagliotti et al. zur Erstlinientherapie des NSCLC, die einen Überlebensvorteil bei nicht plattenepithelialen Karzinomen nachweisen konnte. Am deutlichsten fiel dieser bei Patienten mit Adenokarzinomen aus: Unter einer Behandlung mit Pemetrexed/Cisplatin überlebten sie im Mittel 12,6 Monate, unter Gemcitabin/Cisplatin 10,9 Monate. Mit Pemetrexed als Kombinationspartner gelang es erstmals, das mediane Überleben von Patienten mit fortgeschrittenen nicht plattenepithelialen NSCLC auf über ein Jahr zu verlängern. In beiden Therapiearmen war jeweils ein Drittel der Teilnehmer älter als 65 Jahre. Die Daten belegen: Pemetrexed ist auch bei Patienten höheren Lebensalters effektiv und vergleichsweise gut verträglich.

leben war unter VMP noch nicht erreicht vs. 43,1 Monate unter MP $(\mathrm{p}=0,0008)$ [Mateos MV et al. J Clin Oncol. 2010; 28:2259-66). Besonders hob Salwender die hohe Rate an kompletten Remissionen in der VMP-Gruppe hervor (30vs. $4 \%$; [San Miguel JF et al. N Engl J Med. 2008; 359:906-7]), da auch bei älteren Patienten das Ansprechen positiv mit der Prognose korreliert [Gay F et al. Blood. 2011; 117:3025-31]. Der Proteasom-Inhibitor wurde auch im Rahmen weiterer Therapieregime geprüft: So ließ sich durch eine Bortezomib-haltige Erhaltungstherapie das progressionsfreie Überleben verbessern [Palumbo A et al ASH 2010, Abstract 620]. Weiterhin hat sich Bortezomib auch in Hochdosis-Schemata in der Induktions- und Erhaltungstherapie im Vergleich zu Vincristin bzw. Thalidomid als vorteilhaft bezüglich des Gesamtüberlebens erwiesen.

Symposium im Rahmen des DGHOKongresses 2011 am 2. Oktober 2011 in Basel, Schweiz; Veranstalter: Janssen-Cilag 